\title{
Simulation of Distance Relay for Load Encroachment Alleviation with Agent Based Supervision of Zone-3
}

\author{
Mohamed A. Badr, Nabil H. Abassy Emtethal and N. Abdallah \\ Electrical Engineering Department, Alexandria University, Alexandria, Egypt. \\ eng.mohamedbadr1987@gmail.com, nabil.abbasi@alex.edu.eg, \\ emtethal_1934@yahoo.com
}

\begin{abstract}
Cascaded tripping of power lines due to mal-operation of zone-3 distance relays has been one of the main causes of many previous blackouts worldwide. Encroachment of load into zone-3 characteristics during stressed system operation conditions is a basic factor for such mal-operation of the relays. By improving the operation of zone- 3 , it is possible to prevent mal-operations so that cascaded line tripping can be avoided.For proper study of the behavior of distance relay during faults and load encroachment phenomenon, we must build a model of distance relay, soin this paper a modeling study of distance relay is implemented using MATLAB/Simulink program. However, this model is distinguished from previous modelsthat, examines in detail the third zone of distance relay. Many cases are simulated with changing line loading and fault location to ensure the capability of the relay to detect the fault and thus the maximum loadability limit of distance relay is obtained.In order to prevent cascading events caused by hidden failures in zone-3 relays, agent based relay architectures have been suggested in the recent past. In such architectures each zone-3 relay contains agents that require communication with other agents at various relevant relays in order to distinguish a real zone- 3 event from a temporary overload. In this paper, a local master agent is consulted by all zone- 3 agents before a tripping decision is made. The master agent maintains a rule base which is updated based on the local topology of the network and real time monitoring of the status of other relays and circuit breakers. Cisco Packet Tracer program is used for running communication network simulations. The result of the simulation indicate that the time estimated to send and receive a packet data unit (PDU) message between one relay to anther can satisfy the communication requirement for the proposed scheme with fiber media.
\end{abstract}

Keywords - Distance Relay, Load Encroachment, Hidden Failure, Cascading Blackouts, Agents, Communication, TCP/IP.

\section{INTRODUCTION}

Transmission lines are usually protected by distance relays. The main objective of the distance relaying protection scheme is to isolate a faulted line out of service as soon as possible to minimize the negative effect of the fault on the power grid. The secondary objective of the fault isolation is to minimize the amount of load shedding as a result of the disconnection of lines by distance relay. Distance relays are classified as local primary (zone-1) relays, secondary (zone-2) relays and remote back up (zone3 ) relays [1]. By comparing primary relays with remote backup relays, remote backup relays require longer fault clearing time and also its operation to remove a fault may lead to larger area of load shedding. Therefore the distance relaying protection schemes are designed in such a way that the remote back up relays do not operate unless it is certainly necessary i.e. when both the zone- 1 and zone- 2 relays fail to clear the fault.Worldwide analysis of recent wide area cascading failures has shown that very often these failures were mainly caused by the mis-operation of the third zone of a distance protection relay [2]. The hidden failure, load encroachment and power swing phenomena are the most common problems to solve when dealing with the third zone of distance protection [2]. It is well reported that the majority of blackouts along with their consequences could have been eliminated if the mal operation of distance relays could have been avoided. Zone-3 is especially exposed to load encroachment and power swing, where all these situations can lead to the measured impedance encroachment into the zone-3 area. This results in relay mal-operation and can be a leading factor to a large scale blackout occurrence. Despite 
the fact of zone-3 setting encroachment, the system operational conditions may not be dangerous and in case of load encroachment the load may be permissible due to the transmission lines temporary load ability. In case of stable power swing, after some time the system recovers to its normal operation conditions. The important issue is to distinguish whether the third zone area encroachment is a result of fault and the relay should operate, or it is one from abovementioned situations and the relay decision about tripping should be restrained [3].

Modern study discussed and modeled the characteristics of the Mho relay. In this paper we established a model of distance relaying protection using MATLAB program. In [4] PSCAD/EMTDC Software is used to study the performance of the relay characteristics with different type of faults (L-G, L-L, L-L-G etc.) at different locations. That work presented only the simulation of distance relay during faults. But in our model, we take into account the problems that faced the distance relays during faults and normal operations like the effect of ground resistance during ground faults and the problem of load encroachment respectively. We study the effect of ground resistance by changing the value of ground resistance and studying the effect of it on the impedance of distance relay. Also, we study the problem of load encroachment in our model by increasing the value of load gradually till reach to the maximum loadability limit of distance relay.

A hidden failure is a defect (incorrect relay setting or software or hardware error) in a relay which may go unidentified for a long time and gets excited by another event leading to erroneous removal of circuit elements [5]. Because of hidden failures, zone-3 relays may be extra sensitive to temporary line overloading due to transients, results in unreal fault as a fault in a line and mis-trip even though it is not recognized as a faulty condition by zone-1 or zone-2 relay. At this instance, if power system is operating under stressed conditions, the hidden failure induced zone-3 relay mis-trip may initiate other line trips leading to catastrophic failures like blackouts.

According to the analysis of historical blackouts such as the 1965 Great North-east blackout, the 1977 New York blackout and the 1996 western blackout, North American Electric Reliability Council (NERC) concluded that the mis-operation of zone-3 relays is one of the major causes of cascading outages leading to blackout events [2], [10]. Therefore, Horowitz and Phadke[6] reviewed the distance relaying protection scheme to judge the necessity of the remote back up relays but concluded that the zone- 3 relays cannot be omitted as its elimination will put the power system at risk. Also, according to a report from the latest 2003 blackout in the US [7], many of zone-3 distance relays operated under the overload situation, which further stressed the system thereby causing the cascading blackout in the end. This kind of relay mal-operation may further weaken the system. Zone-3 relays can incorrectly trip a line due to hidden failures [8], [9].

One of the main objectives of smart grid is to prevent these types of mis-operations of power system protection components, by establishing communication between its components. This paper concerns on zone-3 relay mis-operation. In [11] a distributed agent based supervisory scheme is proposed to make zone- 3 relays able to withstand hidden failure induced tripping, facilitated by communication network. In their approach the entire power grid is populated with software components called agents at every relay. Communication architecture is established between hierarchically distinguished master and slave relay agents to help making critical decisions. In order to aid the zone-3 relays to distinguish between a real fault and an unreal fault (due to unexpected loading conditions), in this paper we establish a simple model of communications between relays using TCP/IP protocol to help the distance relaying scheme reducing the zone-3 mis-operation further reducing the cascading outages.

The rest of this paper is organized as follows: section II presents the implementation of distance relays model using MATLAB software. The load encroachment phenomena and loadabilitly limits of distance relays are explained briefly in section III. Section IV describes the agents rules of zone-3 to avoid mis-operation of distance relay, also presents the established model in Cisco Packet Tracer program. Section $\mathrm{V}$ concludes the paper.

\section{MODELING AND SIMULATION OF DISTANCE RELAY}

Fig. 1 shows the basic distance protection scheme of a transmission line. The impedance measurement inputs are the values of three phase current and voltage signals taken from the current transformer 
(CT) and potential transformer (PT) respectively. Three phase current and voltage waveforms must be filtered first to eliminate the harmonic contents which may present due to arcing of the fault. The filtered waveforms are then sampled at a selected sampling frequency before being used digitally inside distance relay. The relays compare the setting impedance with the measured impedance to determine if the fault is inside or outside the protected zone.

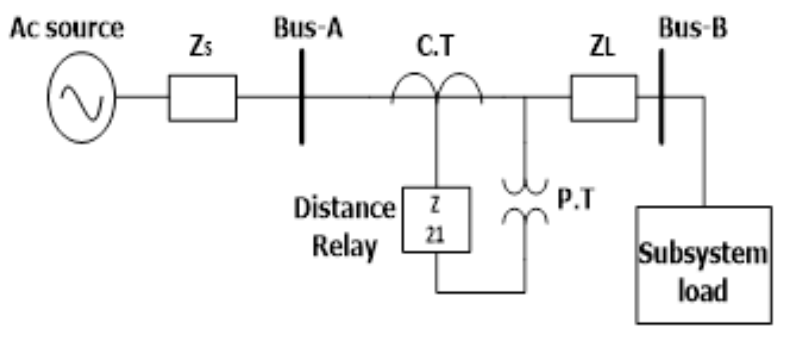

Fig .1. One-line diagram of simulation system

Distance relays are also named impedance relays [12]. They are used to calculate line impedance by measurement of voltages and currents at one single end. For example, as shown in Fig. 2 Mho type distance relays, by comparing the setting value and the measured value the relay can determine if there is a fault or not .Distance relays immediately release a trip signal when the impedance value is inside the zone-1 impedance circle of distance relay. For security protection consideration, the confirmation of a fault occurrence will not be made until successive trip signals are released in one season (faulted section).

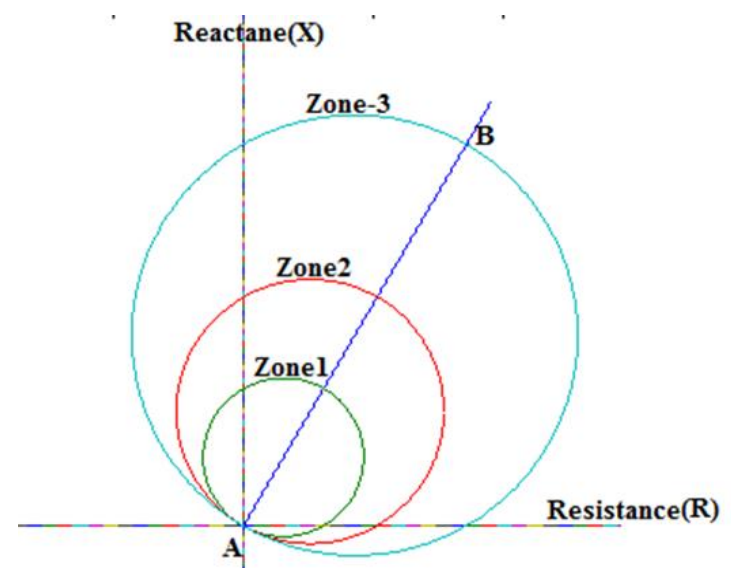

Fig .2. Distance relay of mho characteristic type

Different formulas should be adopted when calculating the fault impedance due to different fault types. Table 1 indicates calculation formula for all of the fault types. [13].

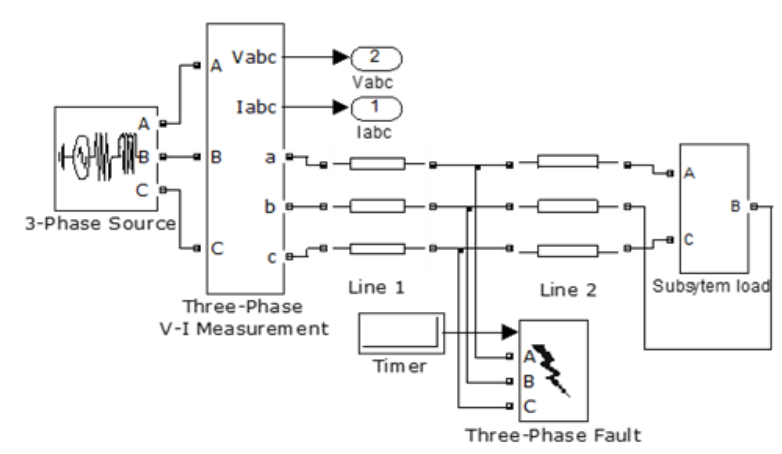

Fig .3. Snapshot of the transmission line and load model

Table 1. Fault impedance formula for different faults

\begin{tabular}{|c|c|}
\hline Fault Type & Formula \\
\hline Phase A to G & $\mathrm{V}_{\mathrm{A}} /\left(\mathrm{I}_{\mathrm{A}}+3 \mathrm{~K}_{0} \mathrm{I}_{0}\right)$ \\
\hline Phase $B$ to $G$ & $V_{B} /\left(I_{B}+3 K_{0} I_{0}\right)$ \\
\hline Phase $C$ to $G$ & $V_{C} /\left(I_{C}+3 K_{0} I_{0}\right)$ \\
\hline Phase A to Phase B & $V_{A B} /\left(I_{A}-I_{B}\right)$ \\
\hline Phase B to Phase C & $V_{B C} /\left(I_{B}-I_{C}\right)$ \\
\hline Phase $\mathrm{C}$ to Phase $\mathrm{A}$ & $V_{C A} /\left(I_{C}-I_{A}\right)$ \\
\hline
\end{tabular}

where,

$A, B$ and $C$ indicate faulty phases, $G$ indicates ground line, VA, VB and VC indicate voltage phasors, IA, IB and IC indicate current phasors, 10 is zero-sequence current, $\mathrm{KO}=$ residual compensation factor where $\mathrm{KO}$ $=(Z 0-Z 1) / 3 Z$, where $Z 0=$ line zero-sequence impedance, $Z 1$ = line positive-sequence impedance.

In this paper in order to get exact simulation results, we must establish accurate network model. Simulink/PSB (power system blockset) is used to create power system model for simulation. Fig. 3 shows the developed model of transmission line and load in MATLAB/Simulink. From this figure, it can be seen that the transmission line is separated into two equal lines. The reason is to simulate a fault at a point along the transmission line where the first line simulates the fault distance from the substation terminal until fault point while the second line simulates the remaining distance from fault point until the end of the transmission line.

When power systems faults occur, the signals may contain high frequency components. As shown in Fig.4, these higher frequency components must be eliminated, so we adopt analogue low pass filters in the simulated systems. After filtration, the only remained fundamental voltage and current waveforms will be passed to FFT blockset. The 
function of FFT blockset is to extract the magnitudes and phase angles of fundamental three phase current and voltage phasors. The FFT blockset performs a Fourier analysis of the input signal over a running window of one cycle of the fundamental frequency of the signal. These magnitudes and phase angles are then used by the fault calculation algorithmblockset to calculate the impedance of the faulted phase.

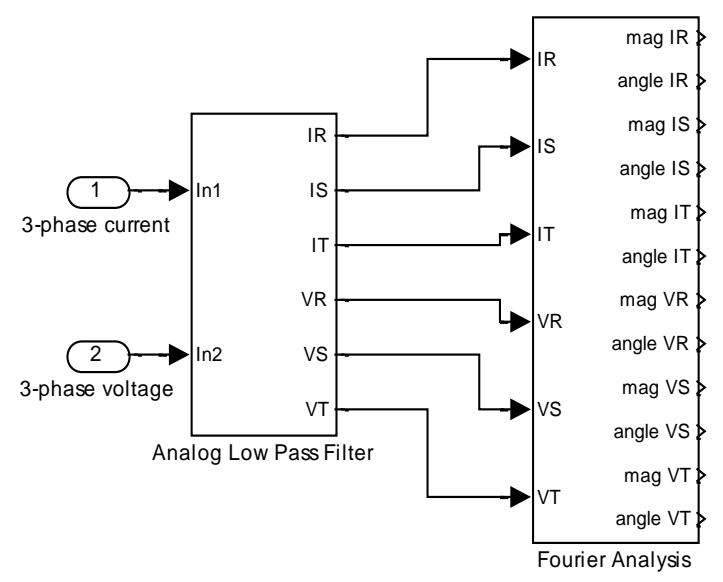

Fig .4. Snapshot of the analysis of 3 phase faulted current and voltage

The parameters of the simulated transmission system are [13]:

Voltage Rating: $132 \mathrm{kV}$ at $50 \mathrm{~Hz}$

Length of Transmission Line: $50 \mathrm{~km}$

Line Impedance:

$Z_{1 \text { line }}=6.8432 \Omega$

$Z_{1}=0.01239+j 0.13630113 \Omega / \mathrm{km}$

$Z_{0}=0.1239+j 0.4089 \Omega / k m$

Fig. 5 shows $\mathrm{R}-\mathrm{X}$ diagram for the simulation of $\mathrm{LG}$ fault at $30 \mathrm{~km}$ from the relay location at fault resistance $(0.1 \Omega)$ and maximum torque angle (MTA) of $60^{\circ}$. From the figure the fault location is found to be at zone- 1 , where zone- 1 setting $=90 \%$ of $Z$ line.

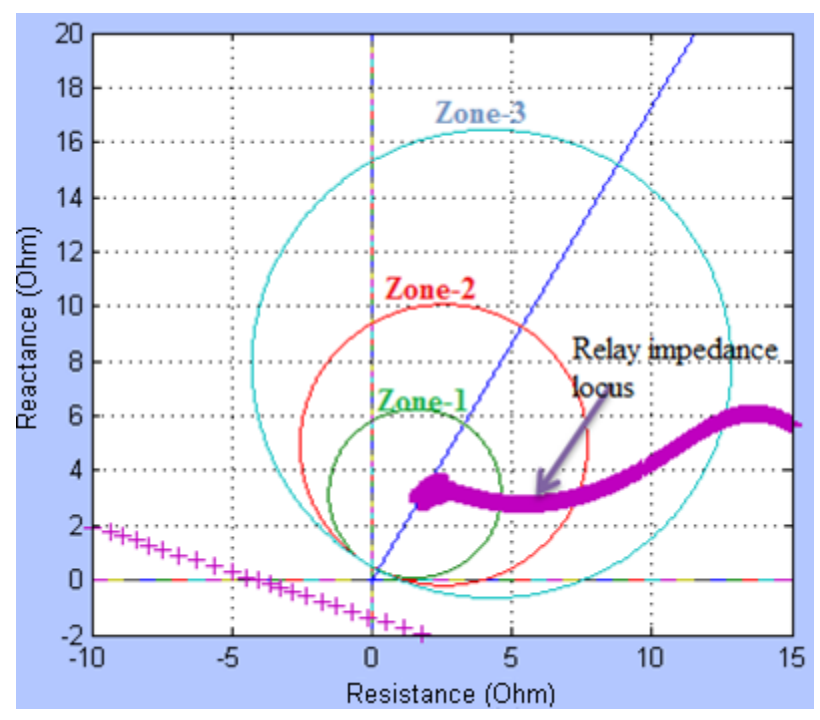

Fig .5. L-G Fault at $30 \mathrm{~km}$ from Bus-A, Zone-1

Fig. 6 shows $\mathrm{R}-\mathrm{X}$ diagram for the simulation of $\mathrm{LG}$ fault at $125 \mathrm{~km}$ from the relay location. From the figure the fault is found to be at zone-3, where zone-3 $=100 \%$ of protected line $+150 \%$ of the next protected line. This figure also shows that there is a variation in the relay impedance locus, where this variation existed only in the beginning of simulation until the relay impedance locus reaches the final locus

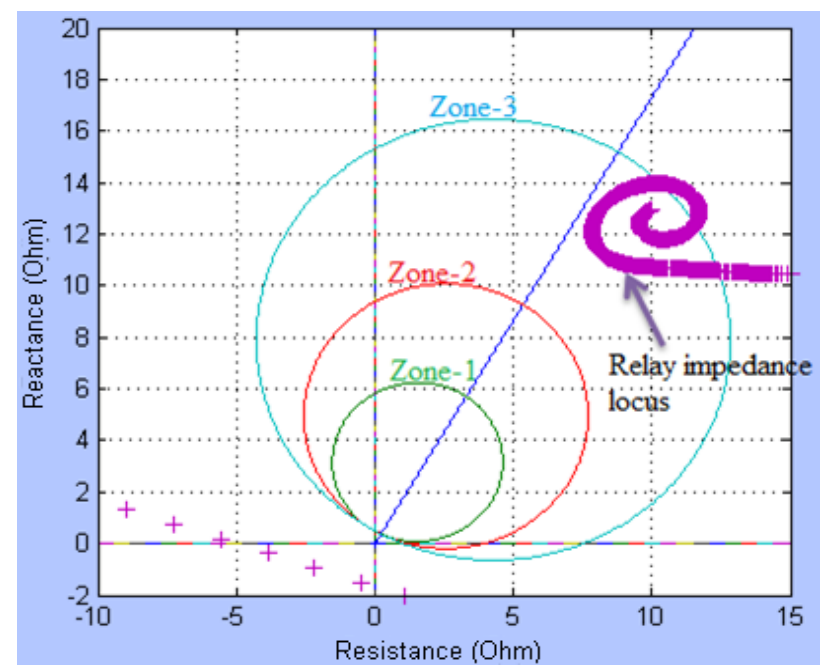

Fig .6. L-G Fault at $125 \mathrm{~km}$ from Bus-A, Zone-3

The reach of the Mho relay is affected the presence of fault resistance as shown in the Fig. 7. AB is the line to be protected, due to fault resistance $B C$. The impedance seen by the relay getting out of the zone.Therefore Mho relay under reaches because of fault resistance [14]. 


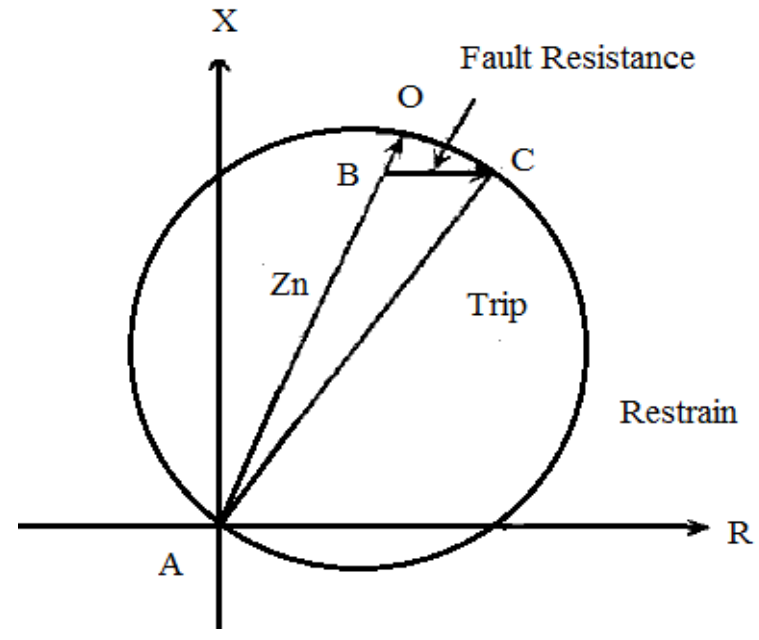

Fig .7. Effect of fault resistance on reach of the relay

Fig. 8 shows $R-X$ diagram for the simulation of $L G$ fault at $30 \mathrm{~km}$ from the relay location. By comparing fig. 5 and fig. 8 it can be noted that the fault resistance has deviated the final point of fault impedance locus far away from zone- 1 reach.

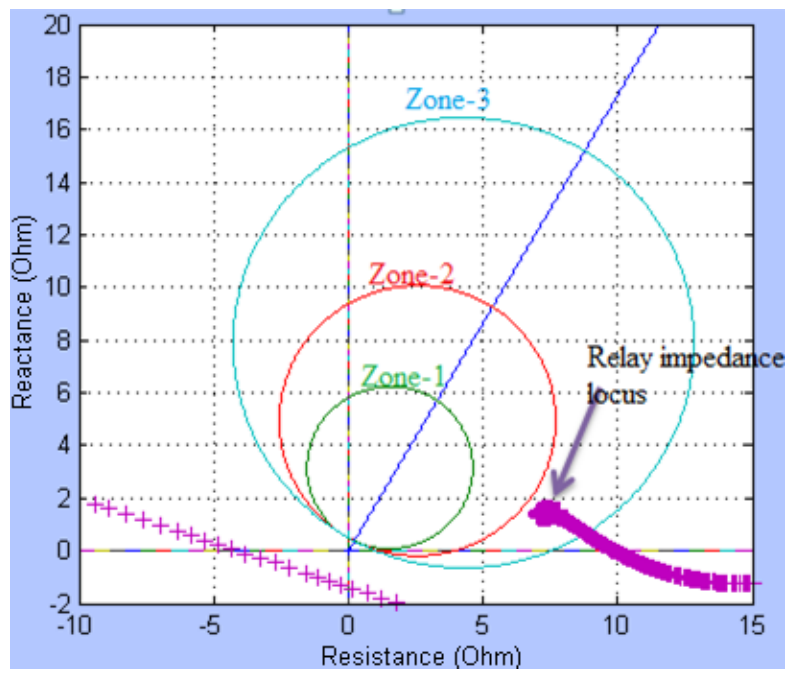

Fig .8. L-G Fault at 30km from Bus-A with $\mathrm{Rf}=10 \Omega$

\section{LOAD ENCROACHMENT AND LOADABILITY LIMITS OF DISTANCE RELAYS}

The relay impedances zones of protection must be selected carefully in order to avoid load encroachment problems. The zone of protection with greater risk is zone-3, since it is the Mho circle with the greatest area and closest proximity to the load impedance. Zone-3 settings are certainly vulnerable to load encroachment conditions during high load and power swings conditions, which can cause the load impedance to travel towards the boundaries of the zone-3 mho circle and cause an undesired trip. Fig. 9 shows the apparent impedance of the load with respect to a distance relay with a forward reaching mho characteristic.

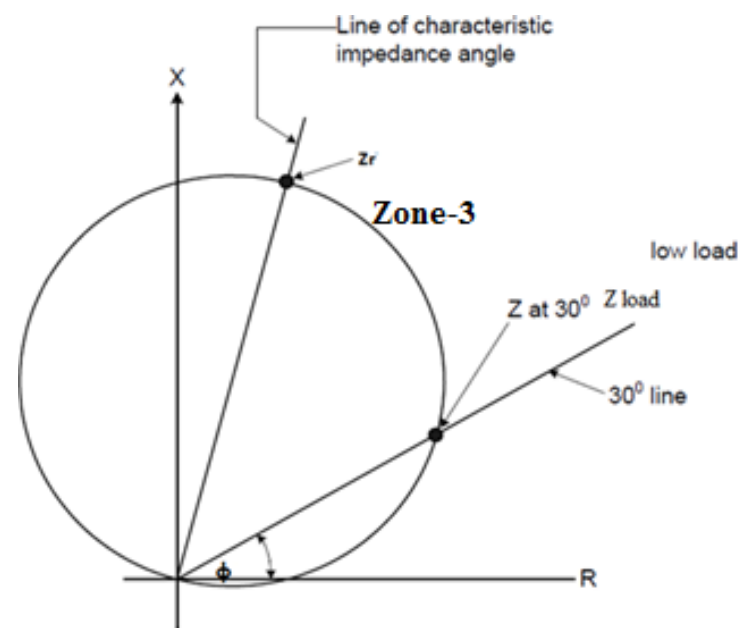

Fig .9. The apparent load impedance w.r.t mho relay

For Mho relay impedance settings, minimum trip MVA is often calculated in accordance with the following formula [15]:

$M V A_{T}=\frac{\left(0.85 \times V_{L L}\right)^{2}}{Z_{r} \times \cos (M T A-\varphi)}$

where,

MVAT = minimum MVA required to trip

$\mathrm{V}_{\mathrm{LL}}=$ nominal line voltage in $\mathrm{kV}$ depressed to $85 \%$ of normal

MTA = maximum torque angle of relay (characteristic impedance angle)

$Z_{r}=$ relay reach in primary Ohms at MTA

$\varphi=$ maximum anticipated phase angle of load .

When using the above calculated loadability limits for planning or operating purposes, it is important that the user be advised whether the limits include any margins [16]. By MVA_TEqu. (1), the minimum MVA required to trip in the proposed model is 618 MVA. Fig. 10 shows the R-X diagram where the load impedance increased to 618 MVA under the normal operating condition. It may be noted that the relay impedance located in the circle of zone- 3 so, the relay will see the increasing of load as a fault and the relay will trip the circuit breaker. By comparing with the value of the calculated minimum MVA required to trip, we will conclude that $618 \mathrm{MVA}$ is approximately the minimum value for the occurrence of the load encroachment phenomenon for this relay. 


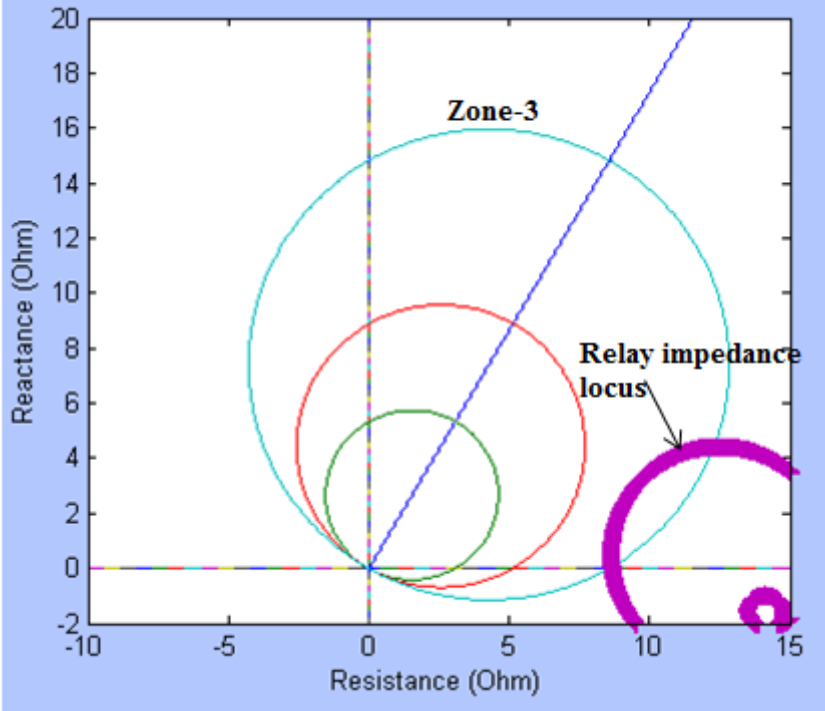

Fig .10. Normal operation at 618 MVA

For distance elements, such as "Mho" characteristic, the susceptibility of the zone to pickup on load generally increases as the reach (impedance setting) is increased. The mho characteristic is most likely to respond to system transient load changes, but may also detect steady-state load - especially when it is heavy and inductive in nature. Alterations in zone characteristics can be made which will reduce the susceptibility of distance zone responding undesirably to a load condition; some of them are outlined as following [16]:

1. Increase of the torque angle.

2. Adoption of a lens characteristic.

3. Use of blinders.

4. Use of load encroachment characteristic.

Options 1, 2, and 3 above, reduce the susceptibility of the relay response to heavy load conditions but reduce the coverage of resistive faults. Option 4 presents the most effective and reliable method of discriminating system faults from heavy load conditions. The load encroachment is a protection feature available in modern line relay packages (for example: [17], [18]) that enables the protection engineer to define custom load regions in forward and reverse direction. Modern study like [19] improves the operation of the distance protection relay by introducing a new blocking algorithm using Dynamic Thermal Line Rating (DTLR) to restrain relay from tripping when conditions in electrical power system allow for it. This reference focused on the possibility of Dynamic Thermal Line Rating usage to prevent distance protection relay from tripping in situations of extreme load conditions and power swing by introducing an additional blocking signal into the distance relay. The blocking signal is based on the DTLR technique monitoring weather conditions and calculating the overhead conductor temperature and actual for ambient weather conditions conductor current limit and the author [19] analyzed this technique by using MATAB/Simulink.

In the implemented model, different values of maximum torque angle for example $\left(60^{\circ}, 75^{\circ}\right.$, and $90^{\circ}$ ) are applied. As shown in Fig. 11, the simulation results show that,to increase line loading (greater than minimum MVA required to trip), the MTA angle can be adjusted on some relays up to 90 degrees. As indicated, such adjustments can increase line loadability as measured along the load apparent impedance line. If the applied relay has the capability of increasing MTA, this method maintains trip dependability while increasing loadability security with minimum cost implications.

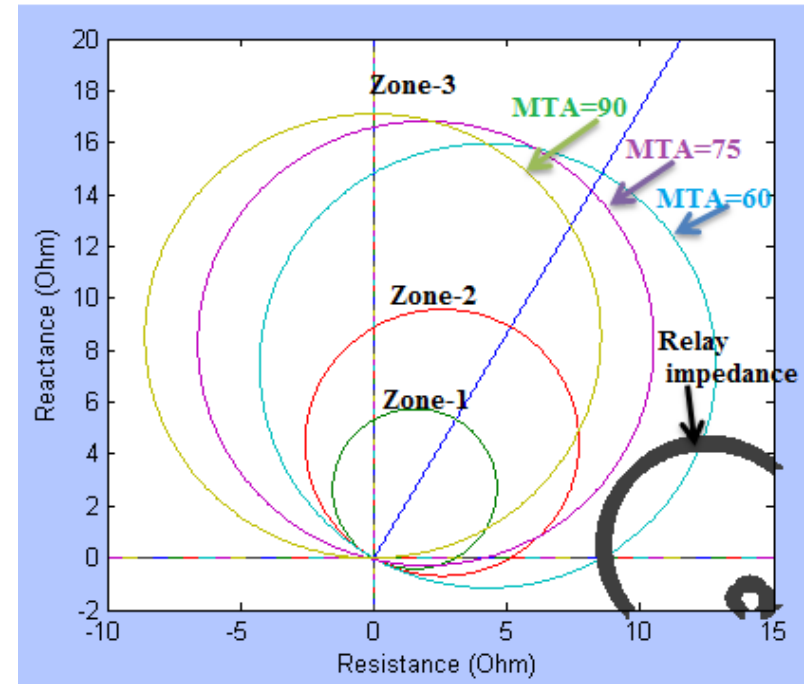

Fig .11. Mho circle torque angle adjustment

\section{AGENT RULES OF ZONE 3 DISTANCE RELAYS TO PREVENT HIDDEN FAILURES AND BLACKOUTS}

\section{A. Model}

Further studies suggested that incorrect operation performed by zone- 3 back up protection relays is due to the presence of hidden failures that may trip a healthy line instead of a faulty line. Such false trips may lead to a sequence of trips resulting in a catastrophic failure like blackout [8]. 
In this paper, a distributed agent based supervisory model is suggested to make zone-3 relays able to distinguish between real faults and false faults specially hidden failures induced tripping, facilitated by the communication network to become an integral parts of the smart grid. Zone-3 relays are often overly sensitive to remote line overloading, and are known to cause mis-trips during cascading failure scenarios. Therefore, providing robustness to zone- 3 relays to minimize the risk of erroneous trips, especially when hidden failuresare existed [8], is an important problem. In the proposed schemes, a grid is populated with agents at each relay, and an agent hierarchy is maintained in master/slave relationship. The communication established between relay agents decreases the probability of erroneous zone- 3 trips thereby preventing them from annoying cascading failure scenarios, and reducing the probability of cascading blackouts. Many studies [20], [21] discussed this issue and presented different approaches and schemes for communications between agents.

It is possible that a fault in a single transmission line can be sensed at least by six relays i.e. zone-1, zone2 and zone- 3 from both ends of the transmission line. Therefore in order to classify a sensed fault as a real fault or an unreal fault, agent of the faulted zone (relay that sense fault) has to communicate with at least five other agents. This can result in huge communication overhead and longer response times. If the total response time is greater than the relay fault clearing times, agent based relay supervision scheme does not serve the intended purpose. Therefore, in order to reduce the response times, agents are hierarchically distinguished as slave agents and master agents. A slave agent associated with a relay is called as "slave agent" and a master agent associated with a relay is called as "master agent".

A slave agent relay records the fault status of its associated relay and reports it to the master agent. Master agent is endowed with high privileges and responsibilities. At any given time, a master agent has the fault status information of all the slave agents reporting to it. Therefore whenever a relay senses a fault, its associated slave relay agent records it and queries the master agent, master agent compares the queried slave relay agent's fault status with the fault statuses of other slave agent relays protecting the same transmission line to classify the fault as a real fault or an unreal fault and respectively acknowledges the queried slave relay agent to trip or not to trip the line out of service [21].

In proposed scheme as shown in Fig. 12, each relay is associated with an agent that has the ability to communicate with other agents in the network. Whenever a relay senses a fault in the transmission line protected by it, its associated agent records it and communicates with other agents protecting the same transmission line in the network to find out if the perceived fault is real fault or unreal fault. If the majority of other relays protecting the same transmission line also sense a fault, classifying it as a faulty condition, master agent can advise its associated relay to trip. On the other hand if the majority of the other relays protecting the same transmission line do not sense a fault, categorizing it as a fault-free condition,master agent advises the relay not to trip. This is because the relay might have sensed a fault due to temporary loading conditions and it is not required for the relay to trip.

In this paper we focus only on the communication between relay agents and estimate the sending and receiving time, and we do not take into account the setting of agents. In this work, to gain better evaluation of communication for the zone-3 supervision scheme we use Cisco Packet Tracer program as a communication tools.

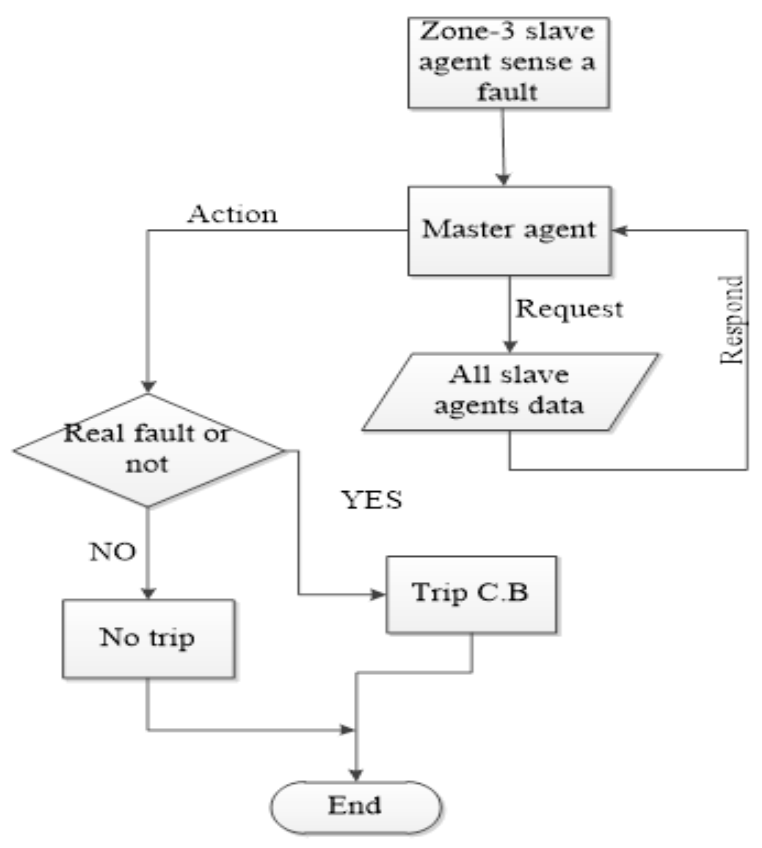

Fig .12. Flow chart of the proposed scheme of agent rules

The master agent has the topology of transmission system within its domain, including the relays and 
breakers. All other agents in the domain are considered as slave agents. In the proposed scheme, as shown in Fig. 13, whenever a zone-3 relay senses a fault in its zone as a reduction in impedance, it queries the domain master agent if any other slave agents associated with the associated set of zone-1 and zone- 2 relays reported any abnormality. The master agent then requests the status of the remaining slave relay agents. Based on the response of the other relays, the master agent can decide if zone-1 or 2 could not clear a fault that zone-3 must act upon. If not, the zone-3 will periodically query master agent until the faulty condition is either cleared or the master agent instructs the zone-3 relay to continue with its trip timer. This will reduce the probability of false trips by zone- 3 relays. The request, response delays of communication must be within the time allowed for zone-3 relays to wait before tripping. The choice of the networking topology, the communication protocol, and the physical media of the network will affect these delays. Also, whether to use the Internet or any other type of networks will also have to be decided.

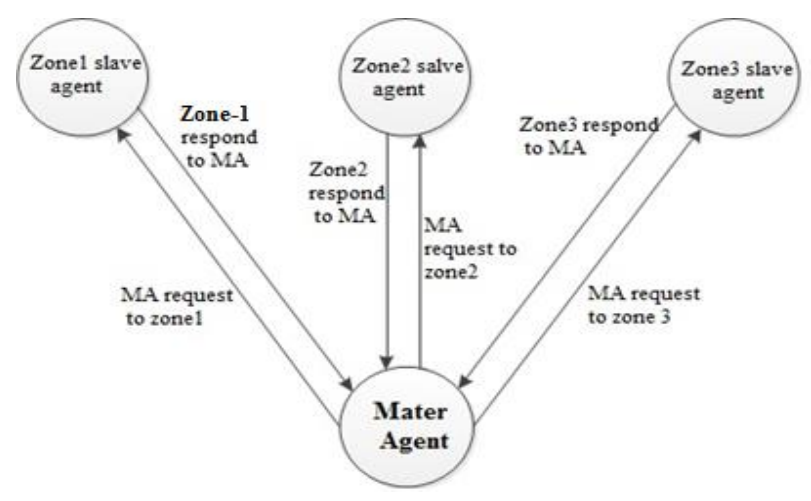

Fig .13. Communications in the proposed scheme

As an illustration, consider a part of the IEEE 14 bus system, as shown in Fig. 14. Consider a fault on the transmission line between buses 10 and 11 in the vicinity of bus 11 , the relays that will sense this fault are, zone- 3 of relays $R(9,15), R(12,12)$ and $R(13,20)$ sense this fault. Ideally the zone- 1 of relays $R(10,17)$ and $R(11,17)$, zone- 2 of relay $R(6,11)$ and zone- 3 of relays $R(9,15), R(12,12), R(13,20)$ should see the fault. Based on the response of the relays, the master agent would locate the fault and would allow the zone3 timers of relays $R(9,15), R(12,12), R(13,20)$ to continue, whereas it would block the zone 3 timer of all other relays $((14,16)$ in this case). If there is any zone-1 or zone-2 relay operation and subsequent circuit breaker operation, resulting in a successful clearing of the fault, the master agent would silence all the zone- 3 timers. Thus the operation of zone- 3 is under the supervision of the master agent. One other expected benefit of this scheme is the identification of hidden failures in these relays. For example, in the above scenario if any of the listed relay pick-ups is not observed or other relays (like $(14,16)$ ) respond, it is an indication of a hidden failure in the relay that has not responded or responded wrongly, in sensing the fault.

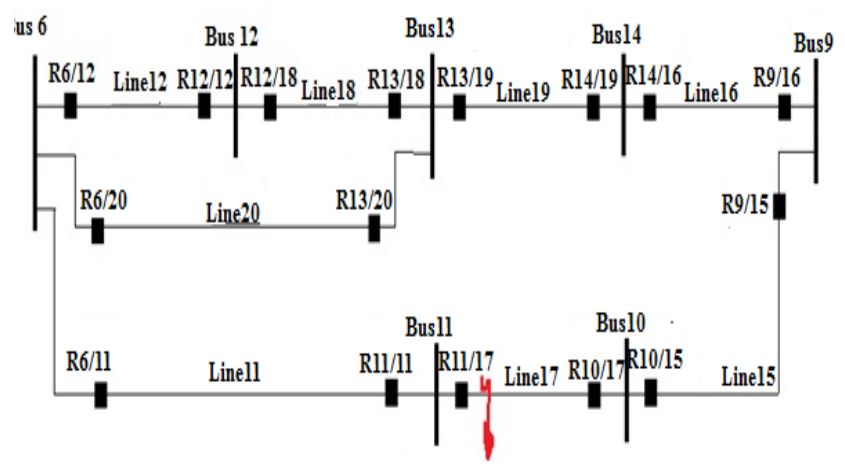

Fig .14. Part of IEEE-14 bus system

Many studies [11], [20] presented different types of communications and response time. In [11] the study was experimented with various communication topologies, protocols, and media to figure out which topology, media, and protocol would make the presented scheme feasible. It is clear that with fiber media, all possible protocols, and topologies will allow enough slack in latency for the studied scheme to work. In [20]three different network topologies and physical media of communication were evaluated to find out the most suitable network topology and physical media that meets the time constraints of zone-3 relay supervision scheme. The networking and optimization simulation results indicate that the proposed scheme can reduce the undesirable zone-3 tripping and thus reduce the probability of occurrence of blackouts. In this paper a model is implemented as follows in the next section to study the time of response between mater agent and slave agents. The results of this model give better response, where the time of response is less that the time gained before in the previous studies.

\section{B. Simulated example:}

We assume each protection relay to be an agent which can communicate with master agent directly. In this way, relays near a bus/substation compose a local area network (LAN) which uses Ethernet as link layer protocol. Then relays can send message from this LAN to other LANs or master agent through 
routers. The links between LANs are fibers media. This simulation actually simulates various TCP/IP based protocols for communication.

We use Cisco Packet Tracer program tool for running communication network simulations. We apply our simulation on 7 buses of IEEE-14 bus system. In this simulation we put a router at each bus and we connect between buses by fiber cables and each router has IP address as shown in Fig. 15. Table 2 shows IP addresses of any cable at the start bus or router to end bus or router.

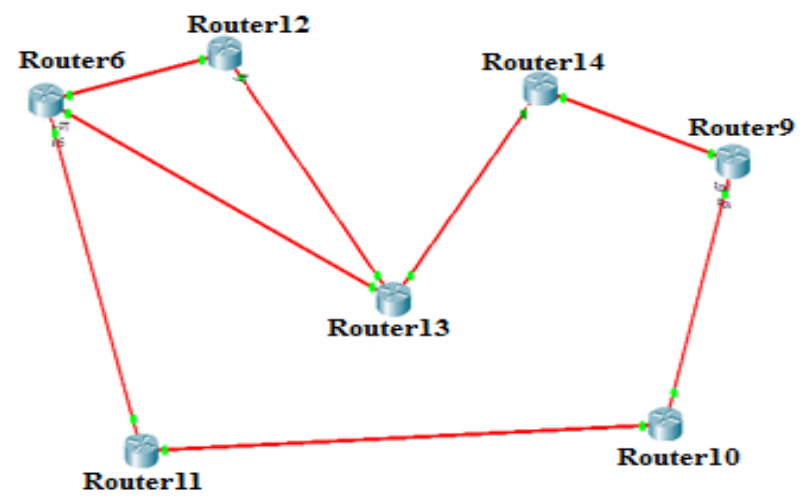

Fig .15. 7 Buses of 14 IEEE bus system in Cisco Packet Tracer program

Table 2. IP addresses for fiber cables

\begin{tabular}{|c|c|c|}
\hline $\begin{array}{c}\text { Bus to bus (router to } \\
\text { router) }\end{array}$ & $\begin{array}{c}\text { Start Bus } \\
\text { (Router) } \\
\text { IP Address }\end{array}$ & $\begin{array}{c}\text { End Bus } \\
\text { (Router) } \\
\text { IP Address }\end{array}$ \\
\hline Router 6-To-Router 11 & 16.0 .0 .1 & 16.0 .0 .2 \\
\hline Router 6-To-Router 12 & 10.0 .0 .1 & 10.0 .0 .2 \\
\hline Router 6-To-Router 13 & 17.0 .0 .1 & 17.0 .0 .2 \\
\hline Router 12-To-Router 13 & 11.0 .0 .1 & 10.0 .0 .2 \\
\hline Router 13-To-Router 14 & 12.0 .0 .1 & 12.0 .0 .2 \\
\hline Router 14-To-Router 9 & 13.0 .0 .1 & 13.0 .0 .2 \\
\hline Router 11-To-Router 10 & 15.0 .0 .2 & 15.0 .0 .1 \\
\hline Router 10-To-Router 9 & 14.0 .0 .2 & 14.0 .0 .1 \\
\hline
\end{tabular}

A master agent at any given instance has the complete knowledge of all relays and breaker status in its region. A slave agent, whenever it senses a fault, sends the fault status of its relay to the master agent. Thus master agent has up-to-date information of all relays/breaker status. At any given time, slave agent has the ability to contact master agent for any required information like fault status of other relays. A zone-3 slave relay agent queries the master agent for the fault status of all relevant relays as soon as it senses a fault due to a drop in impedance of the transmission line it is protecting. Master agent acknowledges by providing the fault status of the respective set of relays. In order to achieve this, master agent needs to have a set of rules of which relays fault status along with its zones of protection, it has to provide for a particular zone-3 slave agent request.

\section{Results:}

Table 3 shows the time required to send a packet data unit (PDU) message between one relay to anther after running the model built in Cisco Packet Tracer program.

Table 3. Time estimated to send a PDU between relays

\begin{tabular}{|c|c|c|c|}
\hline Bus to Bus & $\begin{array}{c}\text { Time } \\
(\mathrm{msec})\end{array}$ & Bus to Bus & $\begin{array}{c}\text { Time } \\
\text { (msec) }\end{array}$ \\
\hline Bus 6 to Bus 12 & 1 & Bus 12 to Bus 6 & 2 \\
\hline Bus 6 to Bus 13 & 1 & Bus 13 to Bus 6 & 2 \\
\hline Bus 6 to Bus 11 & 1 & Bus 11 to Bus 6 & 2 \\
\hline Bus 12 to Bus 13 & 1 & Bus 13 to Bus 12 & 2 \\
\hline Bus 13 to Bus 14 & 1 & Bus 14 to Bus 13 & 2 \\
\hline Bus 9 to Bus 14 & 1 & Bus 14 to Bus 9 & 2 \\
\hline Bus 9 to Bus 10 & 1 & Bus 10 to Bus 9 & 2 \\
\hline Bus 10 to Bus 11 & 1 & Bus 11 to Bus 10 & 2 \\
\hline
\end{tabular}

These results indicate that the delays can satisfy the communication requirement for our scheme with Fiber media.

\section{v. CONCLUSION}

This paper presents undesired operation of distance relay third zone due to load encroachment and hidden failures in power system networks. In this paper a model of distance relay is built using MATLAB/Simulink package, inside the modeling single line to ground fault is chosen to be the fault type and Mho characteristics is chosen to be as the protection scheme. A graphical user interface (GUI) is created using GUI package inside MATLAB for the developed model. The phenomenon of load encroachment is simulated in the established model by changing the value of load until reach the value of zone-3 setting. Also the value of maximum load ability 
limit of distance relay is calculated and simulated using the established model in MATLAB. The problem of cascading events caused by hidden failures in zone-3 is also presented in this paper.We proposed a hierarchically distributed agent based distance relaying protection scheme and use communications between each relay by using IP protocols for communication to reduce the cascading outages like blackouts due to zone- 3 relay undesirable trips. This technique is very helpful to distinguish between real faults and unreal faults.

\section{REFERENCES}

[1] G. Phadke and J. S. Thorp, "Computer relaying for power systems", In second edition, Research Studies Press Ltd and John Wiley \& Sons, 2009.

[2] DamirNovosel, Miroslav M. Begovic, and VahidMadani, "Shedding light on blackout" ,IEEE Power and Energy Magazine, January/February 2004 pp. 32-43.

[3] Thomas L. Baldwin, Magdy S. Tawfik and Miles McQueen, "Contingency Analysis of Cascading Line Outage Events", Power Systems Conference, March 2011.

[4] Swapnil Sharma, JagdishBichve, and YuvrajsinhBharthania, " Performance analysis of Mho relay characteristics using the PSCAD", International Journal of Advance Engineering and Research Development, Volume 2, Issue 1, January -2015,pp. 103-110.

[5] SurachetTamronglak, "Analysis of power system disturbances due to relay hidden failures", Ph.D. Dissertation, Virginia Polytechnic and State University, Blacksburg, Virginia, March 1994.

[6] HOROWITZ S., PHADKE A, "Third zone revisited", IEEE Transaction Power Delivery, 2006, 21, (1), pp. 23-29.

[7] "Final Report on the August 14, 2003 Blackout in the United States and Canada: Causes and Recommendations"[Online]. U.S.-Canada Power System Outage Task Force, 2004 Available:https://reports.energy.gov/BlackoutFina I-Web.pdf.

[8] H. Wang and J. Thorp, " Optimal locations for protection system enhancement: a simulation of cascading outages", Power Delivery, IEEE Transactions on, October 2001,pp. 528-533.

[9] A. G. Phadke, "Hidden failures in electric power systems", International Journal of Critical Infrastructures, January 2004, pp.64-75.

[10] J. Thorp and A. Phadke, "Protecting power systems in the post-restructuring era", Computer Applications in Power, IEEE, 12(1), PP. 33-37, January 1999.

[11] S. Garlapati, H. Lin, S. Sambamoorthy, S. Shukla, and J. Thorp. ,"Agent based supervision of zone 3 relays to prevent hidden failure based tripping", in Smart Grid Communications (Smart Grid Comm), 2010 First IEEE International Conference on, October, 2010, pp.256 -261.

[12] L. C. Wu, C.W. Liu and C. S Chen, "Modeling and testing of a digital distance relay using Matlab/Simulink", IEEE 2005.

[13] MuhdHafizildris, Surya Hardi, MohdZamriHasan, "Teaching distance relay using matlab/simulink graphical user interface", Malaysian Technical Universities Conference on Engineering \& Technology, 2012.

[14] Yashasvi, Vidushi, Ramesh, "Simulation of mho characteristics for transmission line protection using PSCAD" ,IJREAS, Volume 2, Issue 2 ,February 2012.

[15] Power System Relay Commitee Working Group, "Application of overreaching distance relays", 2009.

[16] System Protection and Control Task Force of the North American Electric Reliability Council, "Methods to Increase Line Relay Laod ability," June 7, 2006. [Online] Available:

http://www.nerc.com/docs/pc/spctf/Methods to I ncrease Line Relay Loadability 6-7$06 \% 282 \% 29 . p d f$

[17] G. E. Digital Energy, "D90Plus line distance protection", GE Digital Energy, 1.8 edition, 2012.

[18] Alstom, "MICOM Mho P446 Fast Multifunction Distance Protection Relay", Alstom, 2011. 
[19] VishvadeepJ.Dhawale, Vishal Dake and Prafulla A.Desale, "Blocking the Distance Relay 3rd Zone Operation during Power Swings by DTLR Enhancement in MATLAB/Simulink", International Journal of Electronic and Electrical Engineering. ISSN 0974-2174 Volume 7, Number 7, 2014, pp. 757-765.

[20] ShravanGarlapati, Hua Lin , Andrew Heier, Sandeep K. Shukla, and James Thorp, "A hierarchically distributed non-intrusive agent aided distance relaying protection scheme to supervise Zone 3", Electrical Power and Energy Systems,2013, pp 42-49.
[21] Hua Lin, SanthoshkumarSambamoorthy, SandeepShukla, James Thorp, LamineMili "ADHOC vs. supervisory wide area backup relay protection validated on power/network cosimulation platform", proceedings of the 17th power systems computation conference, Stockholm, Sweden, August 22-26,2011. 